\title{
Engineering of Anti-CD133 Trispecific Molecule Capable of Inducing NK Expansion and Driving Antibody-Dependent Cell-Mediated Cytotoxicity
}

\author{
Jörg U. Schmohl, MD ${ }^{1,2}$ \\ Martin Felices, $\mathrm{PhD}^{3}$ \\ Felix Oh, BS \\ Alexander J. Lenvik, BS 3 \\ Aaron M. Lebeau, $\mathrm{PhD}^{4}$ \\ Jayanth Panyam, $\mathrm{PhD}^{4}$ \\ Jeffrey S. Miller, MD ${ }^{3}$ \\ Daniel A. Vallera, $\mathrm{PhD}^{1}$
}

\section{${ }^{1}$ Department of Therapeutic}

Radiology-Radiation Oncology,

University of Minnesota, Masonic Cancer

Center, Minneapolis, MN, USA,

${ }^{2}$ Department for Hematology and Oncology,

Medicine Department 2, University Hospital

of Tuebingen, University of Tuebingen,

Tuebingen, Germany, ${ }^{3}$ Department of

Medicine, Division of Hematology, Oncology,

and Transplantation, ${ }^{4}$ Department of

Pharmacology, University of Minnesota,

Minneapolis, MN, USA

Correspondence: Daniel A. Vallera, PhD

Department of Therapeutic Radiology-Radiation

Oncology, University of Minnesota,

Masonic Cancer Center,

MMC: 494, Minneapolis, MN 55455, USA

Tel: 1-612-626-6664

Fax: 1-612-626-4842

E-mail: valle001@umn.edu

Received October 13, 2016

Accepted January 30, 2017

Published Online February 20, 2017

\begin{abstract}
Purpose
The selective elimination of cancer stem cells (CSCs) in tumor patients is a crucial goal because CSCs cause drug refractory relapse. To improve the current conventional bispecific immune-engager platform, a 16133 bispecific natural killer (NK) cell engager (BiKE), consisting of scFvs binding FcyRIII (CD16) on NK cells and CD133 on carcinoma cells, was first synthesized and a modified interleukin (IL)-15 crosslinker capable of stimulating NK effector cells was introduced.
\end{abstract}

\section{Materials and Methods}

DNA shuffling and ligation techniques were used to assemble and synthesize the 1615133 trispecific NK cell engager (TriKE). The construct was tested for its specificity using flow cytometry, cytotoxic determinations using chromium release assays, and lytic degranulation. IL-15-mediated expansion was measured using flow-based proliferation assays. The level of interferon (IFN)-y release was measured because of its importance in the anti-cancer response.

\section{Results}

1615133 TriKE induced NK cell-mediated cytotoxicity and NK expansion far greater than that achieved with BiKE devoid of IL-15. The drug binding and induction of cytotoxic degranulation was $\mathrm{CD}_{133^{+}}$specific and the anti-cancer activity was improved by integrating the IL-15 cross linker. The NK cell-related cytokine release measured by IFN-y detection was higher than that of BiKE. NK cytokine release studies showed that although the IFN-y levels were elevated, they did not approach the levels achieved with IL-12/IL-18, indicating that release was not at the supraphysiologic level.

\section{Conclusion}

1615133 TriKE enhances the NK cell anti-cancer activity and provides a self-sustaining mechanism via IL-15 signaling. By improving the NK cell performance, the new TriKE represents a highly active drug against drug refractory relapse mediated by CSCs.

\section{Introduction}

The treatment of cancer stem cells (CSCs) is still a challenge in the management of cancer in humans. Characteristics, such as chemo- and radio-resistance [1], lead to persistence in patients and is a known cause for drug refractory relapse. CD133 has been identified as a frequently expressed marker
Key words

Neoplastic stem cells, Natural killer cell engager,

Antibody-dependent cell cytotoxicity, Interleukin-15, CD133 
Natural killer (NK) cells are large granular lymphocytes that are known for their potential to recognize malignanttransformed and virus-infected cells via a sophisticated repertoire of activating and inhibitory surface receptors that mediate the killing of the target by the insertion of cytotoxic perforin and granzyme toxins. These characteristics make them important effectors of immune surveillance and cancer defense. The lymphoid repertoire of the human body comprises several NK cell subpopulations that differ in their level of receptor expression. The main population capable of eliminating tumor cells is composed of CD $56^{\mathrm{dim}} \mathrm{CD} 16^{\text {bright }}$ cells. The mechanisms for NK-mediated tumor elimination include natural cytotoxicity, antibody-dependent cell-mediated cytotoxicity (ADCC), and the secretion of interferon (IFN)- $\gamma$, which are essential for mediating the anti-tumor activity.

NK cells are activated by interleukin (IL)-15 [6] by binding to the IL-15 receptor complex ( 3 subunits, 2 of them shared with the IL-2 receptor complex). IL-15 mediates the lymphokine- activated killer activity and increases ADCC [6-8]. IL-15 and IL-2 have the ability to mediate the ex vivo expansion of NK cells; up to 20-fold expansion after 2 weeks [9]. This cytokine may have certain therapeutic advantages over IL-2. Studies have shown the benefits of the expansion of allogeneic peripheral blood mononuclear cells (PBMCs) for cancer therapy, but the approach is limited by T cell contamination and the induction of graft-versus-host disease. The potential of IL-15 to induce the expansion and prolonged survival of NK cells in vivo indicates the potential to improve NK cell performance and implicates its use as an effective immunotherapeutic agent [10], particularly if delivered selectively to the NK cell population and the exact site of ADCC in vivo.

Several immunotherapeutic agents were reported to target CD16 [11,12]. All of these show the capability of targeting CD16 target cells selectively. On the other hand, none of these have incorporated IL-15 as a self-sustaining agent capable of stimulating NK expansion.

This study presents a self-sustaining trispecific NK cell engager (1615133 TriKE) that specifically kills CD133+ target cells through the mechanism of ADCC. The construct is composed of a scFv isolated from a hybridoma generated by immunizing against the extracellular domain of CD133. 1615133 TriKE recognizes a framework determinant that crosses species platforms [13]. The construct also contains an anti-CD16 scFv generated in a human phage display library [11] that binds FcGRIII on NK cells to form an immune synapse between the NK cells and CD133 expressing tumor targets, which results in NK cell activation and directed tumor target killing. A human IL-15 encoding cross linker that enhances anti-cancer reactivity significantly was included to provide a priming and expansion signal, leading to an improvement in the NK cell therapeutic potential. This new platform technology has been shown to work with other scFvs targeting a range of cancer antigens and it is hoped that this new iteration will facilitate the targeting of the CSC pool [14-16].

\section{Materials and Methods}

\section{Construction of 1615133 TriKE}

The hybrid gene encoding 1615133 was synthesized using DNA shuffling and DNA ligation techniques. The fully assembled gene (from $5^{\prime}$ end to $3^{\prime}$ end) encoded a $\mathrm{NcoI}$ restriction site; an ATG start codon; anti-human CD16 scFv [17]; a 20 amino acid (aa) segment, PSGQAGAAASESLFVSNHAY; N72D-mutated human IL-15 [18]; the seven amino acid linker, EASGGPE; anti-CD133 scFv [13]; and a NotI restriction site. The resulting 1,884 base pair NcoI/ NotI fragment gene was spliced into the pET28c expression vector under the control of an isopropyl- $\beta$-D-thiogalactopyranoside (IPTG) inducible T7 promoter. DNA sequencing analysis (Biomedical Genomics Center, University of Minnesota, St. Paul, MN) was used to verify that the gene sequence was correct and had been cloned in frame.

\section{Isolation of inclusion body}

For the expression of proteins, the Escherichia coli strain BL21 (DE3) (Novagen, Madison, WI) was used after plasmid transfection. The bacteria were cultured overnight and grown in 800-mL Luria broth containing $50 \mathrm{mg} / \mathrm{mL}$ carbenicillin. When the media reached an absorbance of 0.65 at 600 $\mathrm{nm}$ gene expression was induced via the addition of IPTG (FischerBiotech, Fair Lawn, NJ). After 2 hours, the bacteria were harvested. After a homogenization step in a buffer solution ( $50 \mathrm{mM}$ Tris, $50 \mathrm{mM} \mathrm{NaCl}$, and $5 \mathrm{mM}$ EDTA pH 8.0), the pellet was sonicated and centrifuged. To extract the pellet, $0.3 \%$ sodium deoxycholate, $5 \%$ Triton X-100, $10 \%$ glycerin, $50 \mathrm{mmol} / \mathrm{L}$ Tris, $50 \mathrm{mmol} / \mathrm{L} \mathrm{NaCl}$, and $5 \mathrm{mmol} / \mathrm{L}$ EDTA ( $\mathrm{pH} \mathrm{8.0),} \mathrm{was} \mathrm{used} \mathrm{and} \mathrm{the} \mathrm{extract} \mathrm{was} \mathrm{washed.}$

\section{Refolding and purification}

Refolding and purification processes were described recently $[19,20]$. To refold the proteins, inclusion bodies were dissolved at a 20:1 ratio in a solubilization buffer ( $7 \mathrm{M}$ guanidine hydrochloride, $50 \mathrm{mM}$ Tris, $50 \mathrm{mM} \mathrm{NaCl}, 5 \mathrm{mM}$ ethylenediaminetetraacetic acid, and $50 \mathrm{mM}$ dithiothreitol, $\mathrm{pH}$ 8.0) and incubated for 1 hour at $37^{\circ} \mathrm{C}$. After incubation, the 
pellets were removed by centrifugation and the supernatant was diluted (20-fold) with a refolding buffer $(50 \mathrm{mM}$ Tris$\mathrm{HCl}, 50 \mathrm{mM} \mathrm{NaCl}, 0.8 \mathrm{mM}$ L-arginine, $20 \%$ glycerin, $5 \mathrm{mM}$ EDTA, and $1 \mathrm{mM} \mathrm{GSSG}$ at pH 8.0) and incubated further for 2 days at $4^{\circ} \mathrm{C}$. To remove the buffer, 10 -fold dialysis against $20 \mathrm{mM}$ Tris- $\mathrm{HCl}$ at $\mathrm{pH} 9.0$ in $20 \mathrm{mM}$ Tris- $\mathrm{HCl}$ at pH 9.0 over four column volumes was used. To evaluate the purity, sodium dodecyl sulfate polyacrylamide gel electrophoresis (SDS-PAGE) was performed using Simply Blue life Stain (Invitrogen, Carlsbad, CA). The size of 1615133 was approximately 66,680 Da.

\section{Tissue culture}

The following cell lines were obtained from the American Type Culture Collection: colorectal carcinoma cell line, Caco2; and Burkitt lymphoma cell line, Raji. The Caco-2 cell line was grown in monolayers [21] using RPMI supplemented with $20 \%$ fetal bovine serum (FBS) and $2 \mathrm{mmol} / \mathrm{L} \mathrm{L}$-glutamine. Raji was grown in suspension using Eagle's minimum essential medium supplemented with 20\% FBS and glutamine. Both cell lines were incubated at a humidified atmosphere containing $5 \% \mathrm{CO}_{2}$ at a constant $37^{\circ} \mathrm{C}$. When the adherent cells were more than $90 \%$ confluent, they were passaged using trypsin-EDTA for detachment. For the cell counts a standard hemocytometer was used. Only those cells with a viability $>95 \%$ were used for the experiments, as determined by trypan blue exclusion.

\section{Isolation of NK cells and purification}

To isolate the PBMCs, a histopaque gradient (SigmaAldrich, St. Louis, MO) and SepMate tubes (Stemcell Technologies, Vancouver, Canada) were used. Adult blood was derived from healthy volunteers (Memorial Blood Center, Minneapolis, MN). To enrich the NK cells, magnetic beads (Stemcell Technologies) were used according to the manufacturer's protocol by performing a negative selection. The purity was determined by flow cytometry. The samples were obtained after informed consent and in accordance with the University of Minnesota human subjects Institutional Review Board and the Declaration of Helsinki.

\section{Proliferation assay}

The PBMCs or enriched NK cells from healthy donors were labeled with a proliferation dye (CellTrace Violet Cell Proliferation Dye, Invitrogen) according to the manufacturer's protocol. After staining, the effectors were cultured with $50 \mathrm{nM}$ of the respective drugs (anti-CD16 scFv, antiCD133 scFv, 16133 bispecific NK cell engager (BiKE), and 1615133 TriKE; a targeted toxin consisting of an anti-CD22 and anti-CD19 scFv linked to a diphtheria toxin [DT2219]; or National Cancer Institute [NCI] derived IL-15 alone). After incubation for 7 days in a humidified atmosphere containing $5 \% \mathrm{CO}_{2}$ at $37^{\circ} \mathrm{C}$, the cells were harvested, stained for their viability with Live/Dead reagent (Invitrogen), and surface stained for anti-CD56 PE/Cy7 (BioLegend, San Diego, CA) and anti-CD3 PE-CF594 (BD Biosciences, Franklin Lakes, NJ) to gate on the viable $\mathrm{CD} 3^{-} \mathrm{CD} 56^{+}$population. Data analysis was performed using FlowJo software ver. 7.6.5. (Flowjo Enterprise LCC, Ashland, OR) and proliferation was characterized using the expansion index readout. The formula for the expansion index is described elsewhere [22].

\section{CD107a degranulation assay}

Flow cytometry assays to quantify the lytic degranulation via CD107a surface expression and intracellular IFN- $\gamma$ presence were reported previously [23]. The PBMCs were incubated over-night $\left(37^{\circ} \mathrm{C}, 5 \% \mathrm{CO}_{2}\right)$ in RPMI 1640 media supplemented with $10 \%$ fetal calf serum (RPMI-10). The positive control was supplemented further with $10 \mathrm{ng} / \mathrm{mL}$ recombinant IL-12 (Peprotech, Rocky Hill, NJ) and 100 ng/mL IL-18 (R\&D Systems, Minneapolis, MN). PBMCs were resuspended with noted targets or media after several washing steps with RPMI-10, the cells were then exposed to $50 \mathrm{nM}$ of 1615133 TriKE or the other drugs (16133 BiKE, NCI derived IL-15, anti-CD16 scFv, anti-CD133 scFv) and incubated for 10 minutes at $37^{\circ} \mathrm{C}$ with $5 \% \mathrm{CO}_{2}$. Fluorescein isothiocyate (FITC)-conjugated anti-human CD107a monoclonal antibody $(\mathrm{mAb})$ (lysosomal-associated membrane protein 1) (BD Biosciences, New Jersey, CA) was then added and incubated for 1 hour. After incubation, GolgiStop (1:1,500, BD Biosciences, San Jose, CA) and GolgiPlug (1:1,000, BD Biosciences, San Jose, CA) were added for 3 hours $\left(37^{\circ} \mathrm{C}, 5 \%\right.$ $\mathrm{CO}_{2}$ ). After the washing steps with $1 \times$ phosphate buffered saline, the cells were stained with PE/Cy 7-conjugated antiCD56 mAb, APC/Cy 7-conjugated anti-CD16 mAb, and PE-CF594-conjugated anti-CD3 mAb (BioLegend). The cells were then incubated for 15 minutes at $4^{\circ} \mathrm{C}$, washed and fixed with $2 \%$ para-formaldehyde. After the fixing step, the cells were exposed to permeabilization buffer (BD Biosciences, San Jose, CA) and incubated with Pacific Blue-conjugated antihuman IFN- $\gamma$ (BioLegend) for 20 minutes. The cells were finally washed and evaluated by fluorescence-activated cell sorting analysis using a LSRII flow cytometer (BD Biosciences, San Jose, $\mathrm{CA}$ ) gating on $\mathrm{CD} 6^{+} \mathrm{CD}^{-}$cells.

\section{8. ${ }^{51} \mathrm{Chromium}$ release cytotoxicity assay}

The Caco- 2 cells were labeled with $1 \mu \mathrm{Ci}$ of ${ }^{51} \mathrm{Cr}$ per $1 \times 10^{5}$ target cells at $37^{\circ} \mathrm{C}, 5 \% \mathrm{CO}_{2}$ for 1 hour. After washing to remove the excess ${ }^{51} \mathrm{Cr}$, the Caco- 2 cells were added to a $96-$ 
well round-bottom plate $\left(5 \times 10^{3}\right.$ cells). The resting PBMCs were treated with 1, 5, and $10 \mathrm{nM} 1615133$ TriKE; $10 \mathrm{nM}$ $16133 \mathrm{BiKE}$; or $10 \mathrm{nM}$ anti-CD133 or anti-CD16 scFv at an effector:target (E:T) ratio ranging from 0.08:1 to 20:1. The amount of ${ }^{51} \mathrm{Cr}$ released corresponds to the target cell death and was measured using a gamma scintillation counter. The percentage target cell lysis was calculated using the following formula: [(experimental lysis-spontaneous lysis)/ (maximal lysis-spontaneous lysis) $] \times 100$. To determine the maximal lysis, the ${ }^{51} \mathrm{Cr}$-labeled target cells were treated with $3 \%$ Triton $\mathrm{X}$ for 4 hours.

\section{Binding/blocking assay}

To evaluate the drug binding, $4 \times 10^{5}$ Caco- 2 cancer cells were washed and incubated in $4^{\circ} \mathrm{C}$ with $1,5,10,50,100,200$, and $500 \mathrm{nM}$ of FITC-labeled 1615133 TriKE for 30 minutes. For blocking, $200 \mathrm{nM}$ of an anti-CD133 scFv was added to the same concentrations of the FITC labeled 1615133 and incubated for 30 minutes at $4^{\circ} \mathrm{C}$. After washing, the staining intensity was evaluated using an LSRII flow cytometer (BD Biosciences, San Jose, CA).

\section{Statistical analyses}

The data are presented as the mean \pm standard deviation. For statistical evaluation a Student's t test was used to compare two groups, whereas one-way analysis of the variance (ANOVA) was used for multiple comparisons. Data analysis and presentation were performed using Graphpad Prism 5 (GraphPad Software, Inc., La Jolla, CA).

A
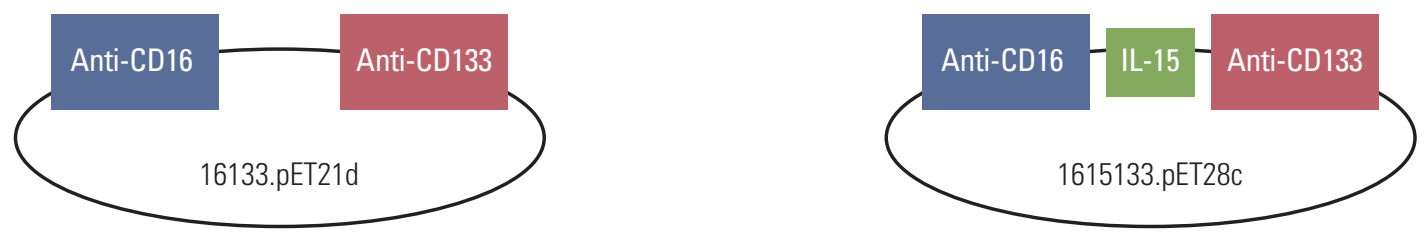

C
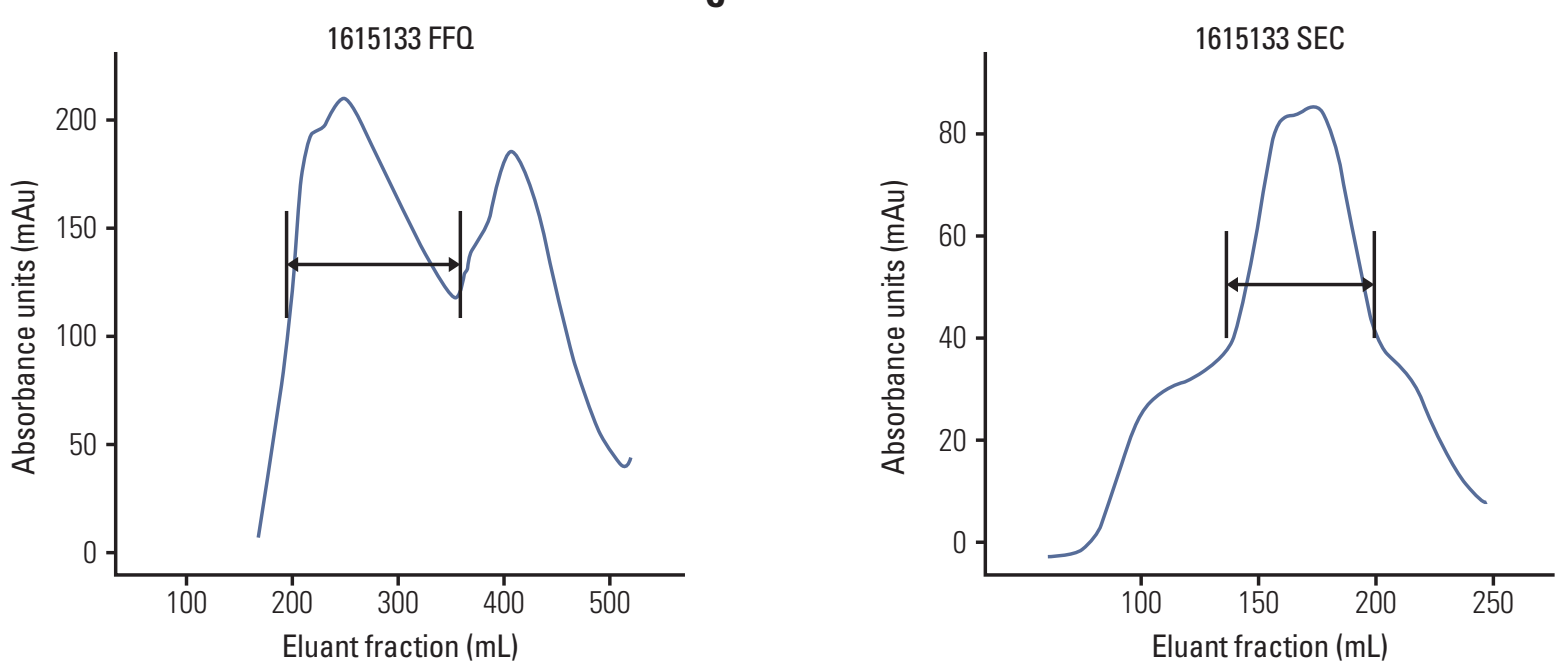

Fig. 1. Construction and purification. (A) 16133 BiKE platform was modified to produce a NK engager capable of immune expansion. (B) A modified IL-15 crosslinker was incorporated between the $\mathrm{V}_{\mathrm{L}}$ and $\mathrm{V}_{\mathrm{H}}$ fragment of anti-CD16 and anti-CD133 scFV forming an IL-15 TriKE (1615133). (C) TriKE trace from the first ion exchange purification column, fast flow sepharose $\mathrm{Q}$ (arrow marks appropriate size range). (D) TriKE trace data from the eluent from the second size exclusion column (arrow marks the appropriate size range). (Continued to the next page) 


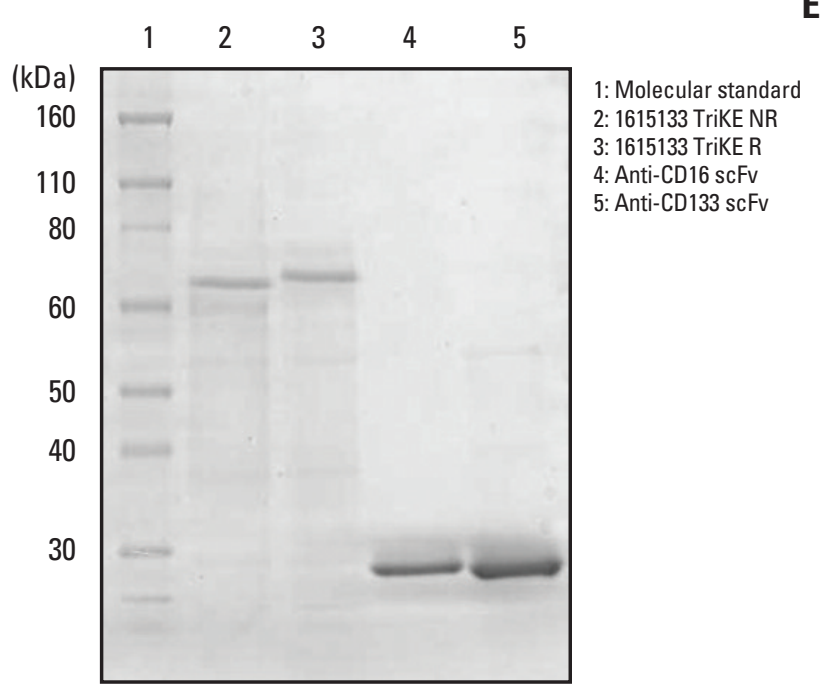

Fig. 1. (Continued from the previous page) (E) The drug is $>90 \%$ pure, as determined by sodium dodecyl sulfate polyacrylamide gel electrophoresis. The size is approximately $66,680 \mathrm{Da}$, as shown by the molecular weight standards in lane 1 (molecular standard). Lane 2 is TriKE non-reduced (1615133 TriKE NR). Lane 3 is TriKE reduced (1615133 TriKE R). For size comparison, lanes 4 and 5 are anti-CD16 and anti-CD133 scFv, respectively. BiKE, bispecific natural killer cell engager; NK, natural killer; IL-15, interleukin 15; scFV, single chain variable fragment; TriKE, trispecific natural killer cell engager.

\section{Results}

\section{1615133 production and purification}

To produce a self-sustaining hybrid NK cell engager, the 16133 BiKE platform (Fig. 1A) was altered with a modified IL-15 crosslinker incorporated between the two scFv constructs forming 1615133 TriKE (Fig. 1B). The new construct contained DNA fragments from the $\mathrm{V}_{\mathrm{H}}$ and $\mathrm{V}_{\mathrm{L}}$ regions of an anti-CD16 scFv, an N72D-modified IL-15 flanked with a 20 aa segment and the 7 aa linker EASGGPE, and the $V_{H}$ and $V_{L}$ regions of an anti-CD133 scFv. Fig. 1C presents the absorbance tracing from the FFQ ion exchange column as the first phase in drug purification. The first major peak eluted from the column represents the drug. Size exclusion column purification (Fig. 1D) and SDS-PAGE gel (Fig. 1E) with Coomasie Blue staining showed high purity (over 90\%) and a protein size of $66,680 \mathrm{Da}$.

\section{Activity of the IL-15 moiety}

The proliferation induced by the IL-15 moiety of the TriKE was measured by CellTrace dye dilution in viable NK and T cell populations. When the donor PBMCs were exposed to 1615133 TriKE or 16133 BiKE, only the TriKE group induced proliferation (Fig. 2A). Importantly, as shown with previous molecules [14,20], the T cells displayed minimal proliferation after 7 days of drug exposure, suggesting that the expansion was mostly restricted to NK cells (Fig. 2B). A comparison with other control agents, including anti-CD16 scFv, antiCD133 scFv, DT2219 (a targeted toxin consisting of an antiCD22 and anti-CD19 scFv linked to a diphtheria toxin), and NCI derived IL-15, showed that only 1615133 TriKE and NCI IL-15 induced proliferation. The other controls showed only the base level proliferation of NK cells (Fig. 2A). To determine the potential of 1615133 TriKE to induce prolonged survival, the purified NK cells were incubated for 7 days with 1615133 or 16133 BiKE. A reactive dye was used to quantify cell death in the different treatment groups. The TriKE group showed a significantly larger amount of live cells, which do not incorporate the reactive dye, compared to BiKE (Fig. 2C). Overall, the results indicate that IL-15 present in 1615133 TriKE induced NK cell proliferation and prolonged their survival.

\section{Activity and specificity of lytic degranulation}

The potential of TriKE to induce lytic degranulation was assessed by examining the induction of CD107a expression on NK cells. As a representative of a CD133 positive cancer population, PBMC were exposed to Caco-2 colorectal carcinoma cells and the coculture was treated with $16133 \mathrm{BiKE}$, 1615133 TriKE, NCI derived IL-15, anti-CD16 scFv, and antiCD133 scFv. Representative gating procedure is shown in Fig. 3A-C and expression is estimated in Fig. 3D. BiKE and TriKE showed significantly enhanced NK cell related degranulation compared to the controls $(p<0.001)$. In Fig. 3E, Raji cells were used as a negative control because they have no CD133 expression. Only supraphysiologic IL-12/ IL-18 stimulation, which did not include targets, showed enhanced degranulation here $(\mathrm{p}<0.001)$, but the level did not appear to be biologically relevant. The data indicate that specific NK cell lytic degranulation occurs only in the presence of CD133-expressing targets.

\section{Specificity of binding and activity of the TriKE}

Standard ${ }^{51} \mathrm{Cr}$ release assays were performed to evaluate the functional activity of 1615133 TriKE in the actual killing of tumor targets, rather than just measuring NK cell degranulation. To determine the effects of incorporating IL-15 into 


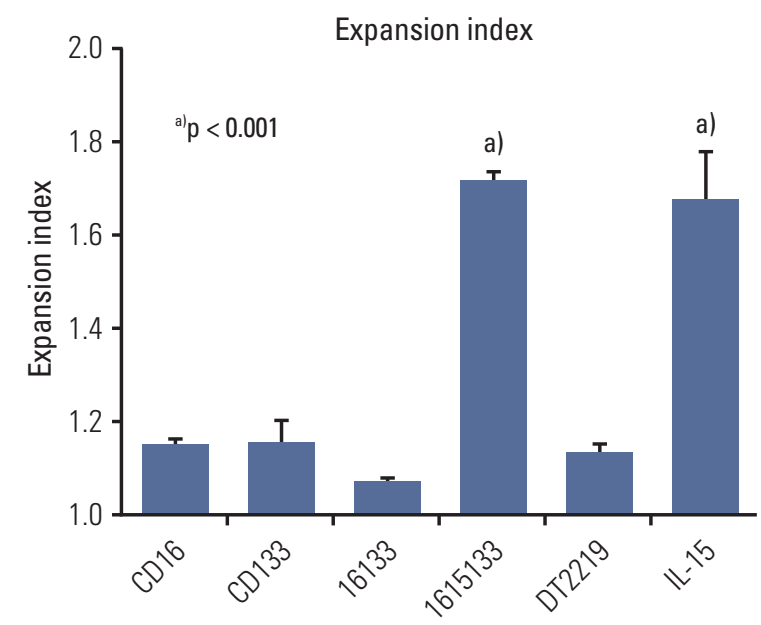

B
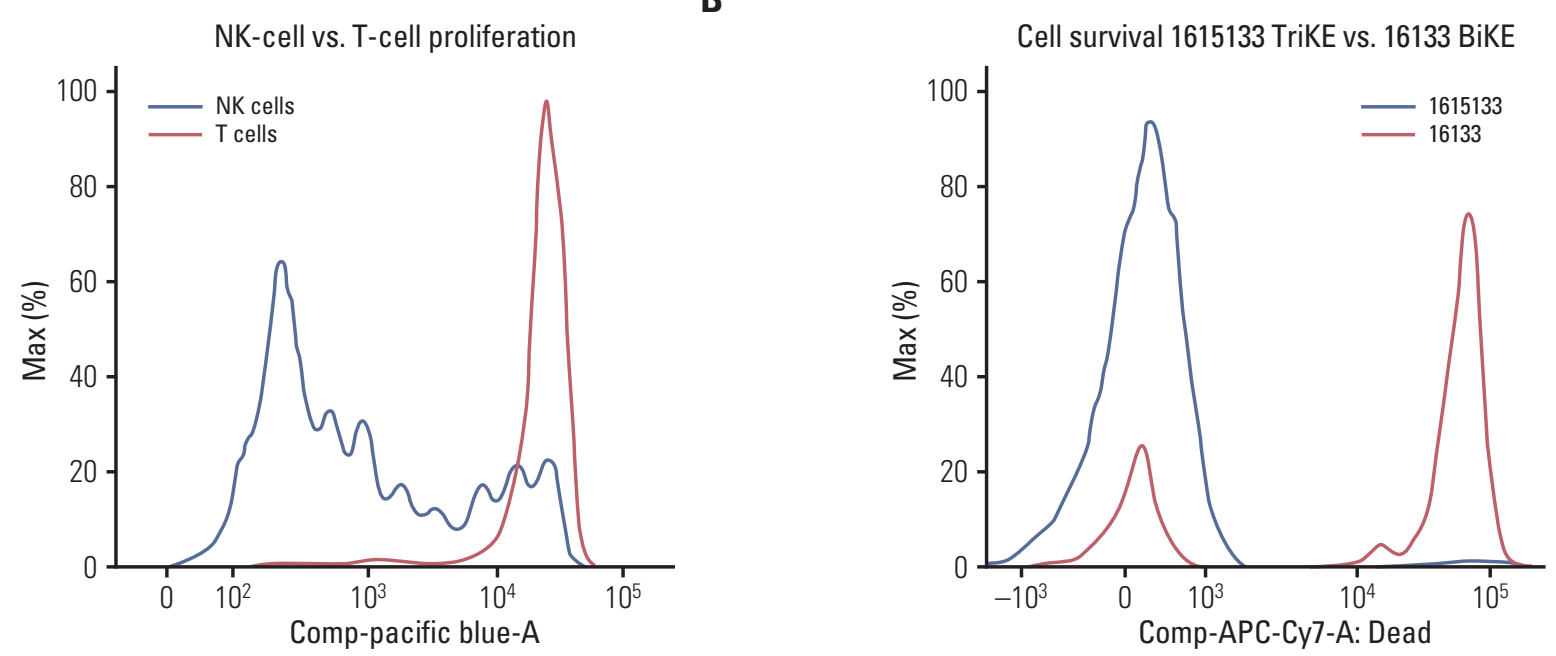

Fig. 2. Expansion and survival. Purified NK cells were exposed to anti-CD16 scFv (CD16), anti-CD133 scFv (CD133), 16133 BiKE, 1615133 TriKE, DT2219 (a targeted toxin consisting of an anti-CD22 and anti-CD19 scFv linked to a diphtheria toxin), and National Cancer Institute-derived interleukin 15 (IL-15). The graph shows data in both raw histogram form (indicating multiple division cycles) and as a formal calculation of the expansion index. (A) As seen after the evaluation of the expansion index, only TriKE and IL-15 increased proliferation significantly (labeled with the letter a) $(n=5)$. Expansion index was calculated using Flowjo software according to the formula: expansion index $=(1-\mathrm{PF}) /(1-\mathrm{Dil})$ (where $\mathrm{PF}=$ fraction of the original population divided at least once during the culture period and Dil=percentage of cells in the final population that have divided), for each group [22]. The significance was estimated by one-way ANOVA and presented with standard deviation. (B) Representative histogram illustrates that after gating on NK cells and T cells, a typical proliferative pattern was visible only in NK cells and not in T cells. (C) Purified NK cells were exposed to 1615133 TriKE and 16133 BiKE and incubated for 7 days. The representative histogram illustrates an impressively larger number of live cells with TriKE compared to the construct without the IL-15 moiety. NK, natural killer; scFV, single chain variable fragment; BiKE, bispecific natural killer cell engager; TriKE, trispecific natural killer cell engager; IL-15, interleukin 15. 
A

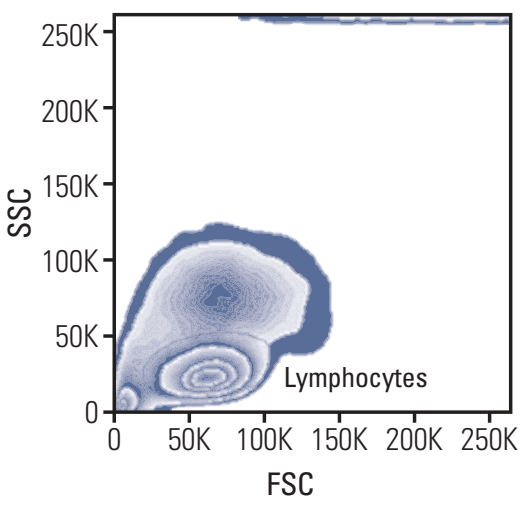

B

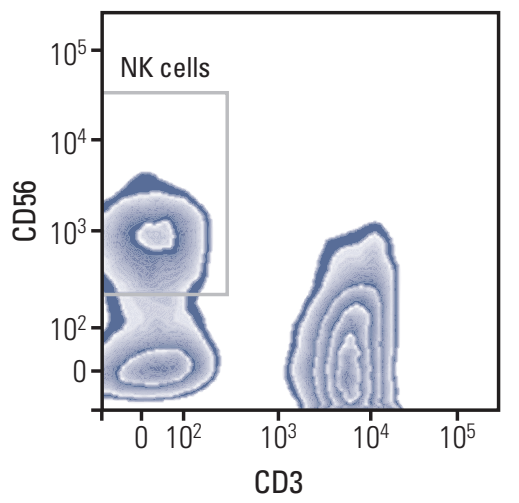

C

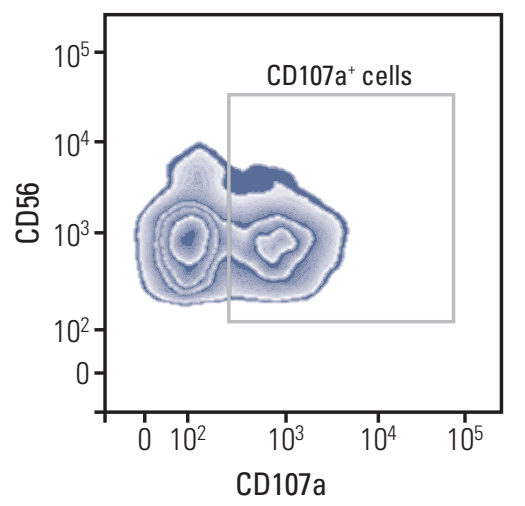

D
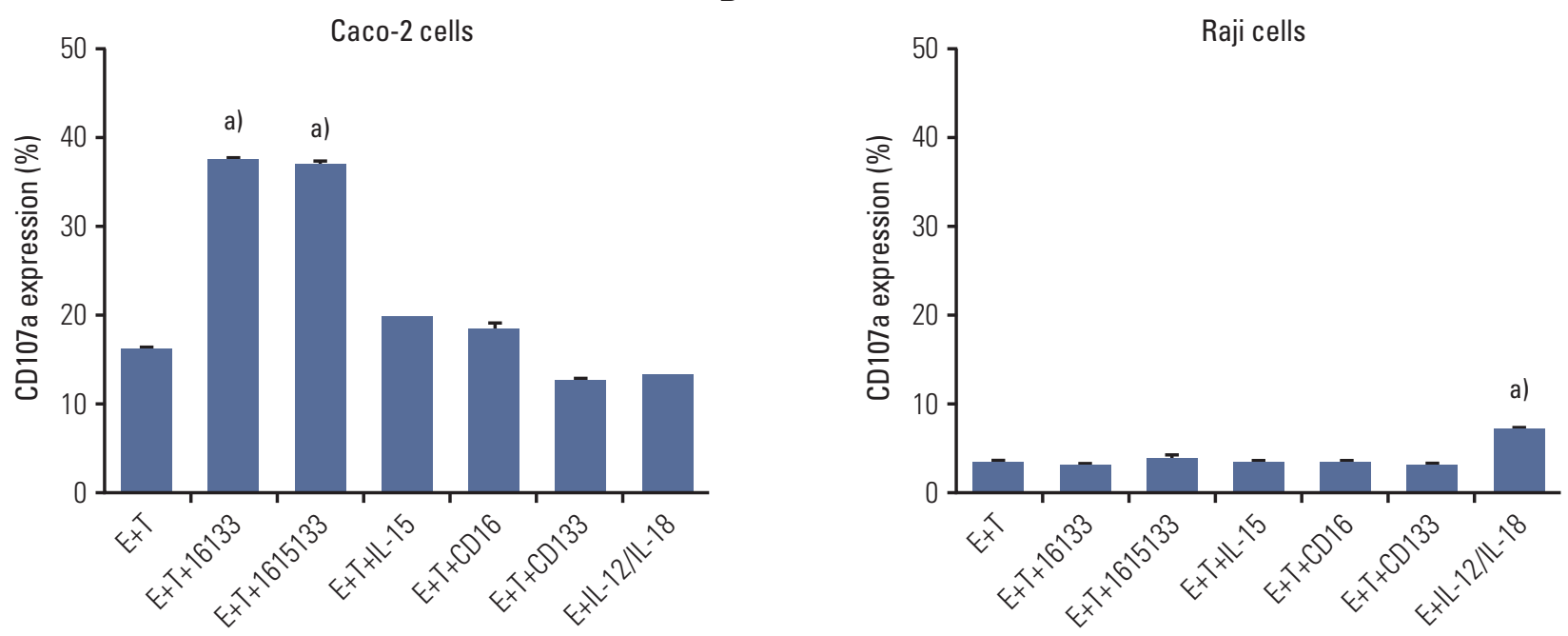

Fig. 3. Induction of the Degranulation of 1615133 TriKE. (A-C) Gating strategy is shown with the representative Zebraplots of effectors and Caco-2 target cells with 1615133 exposure. (D, E) CD133 ${ }^{+}$Caco-2 and CD133- Raji cells were exposed to peripheral blood mononuclear cells and 16133 BiKE, 1615133 TriKE, National Cancer Institute-derived IL-15 (IL15), antiCD16 scFv or anti-CD133 scFv (CD133). The positive control contained peripheral blood mononuclear cells and supraphysiologic concentrations of IL-12 and IL-18 (IL-12/IL-18). With Caco-2 cells the samples with 16133 BiKE and 1615133 TriKE showed significantly higher CD107a expression compared to the controls (noted in graph). For Raji cells, this was only obvious for the IL-12/IL-18 control. The graphs show the pooled data of CD107a expression for each of the groups $(\mathrm{n}=3)$. The significance was estimated with one-way ANOVA and presented with the standard deviation. ${ }^{a)} p<0.001$. TriKE, trispecific natural killer cell engager; BiKE, bispecific natural killer cell engager; IL, interleukin; SSC, side scatter; FSC, forward scatter; NK, natural killer; E, effector; T, target.

the 16133 scaffold, the cytotoxicity was evaluated using NK cells of two separate donors and Caco-2 tumor targets at different E:T ratios (20:1, 10:1, and 5:1) and the differences in activity among 1615133 TriKE, $16133 \mathrm{BiKE}$, anti-CD16 scFv, anti-CD133 scFv, and no drug treatment were compared (Fig. 4A and B). Owing to baseline variations, the repro- ducibility was ensured by repeating the assays with different donors. Killing of the Caco-2 targets was elevated in the TriKE samples compared to the controls, but in the CD107a flow cytometric assays, the level of NK cell degranulation was not significantly higher than in the BiKE group. Furthermore, higher NK cell levels, denoted by the higher E:T ratios, 
A

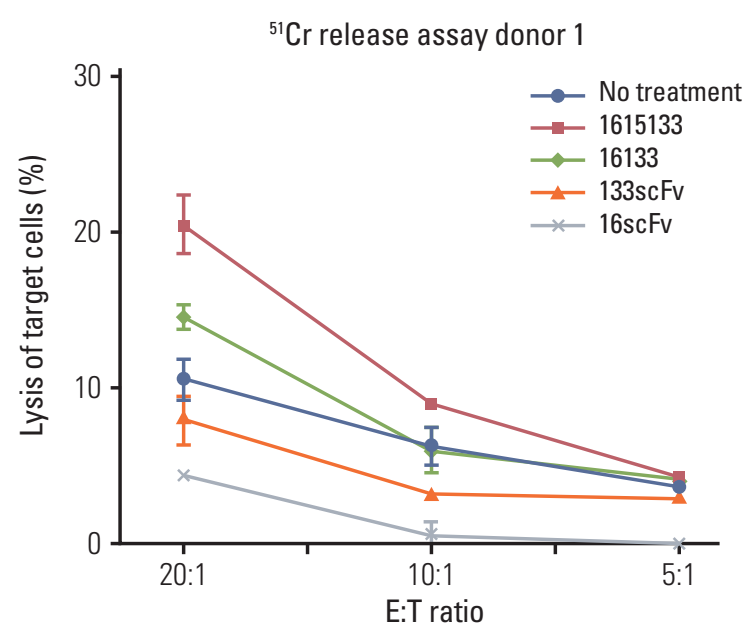

C

Dose/E:T ratio

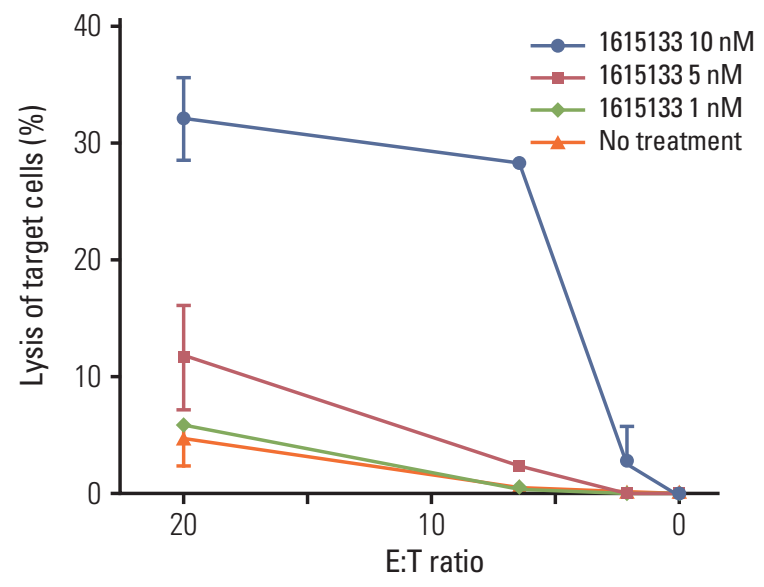

${ }^{51} \mathrm{Cr}$ release assay donor 2

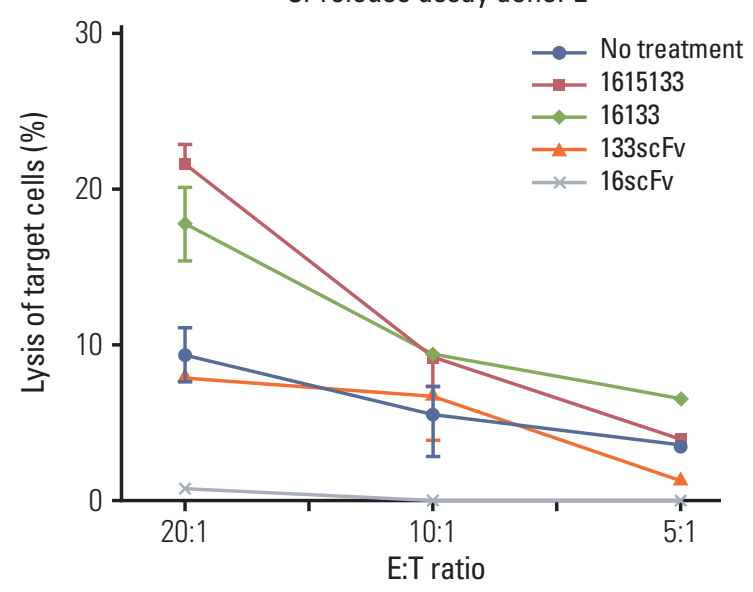

D

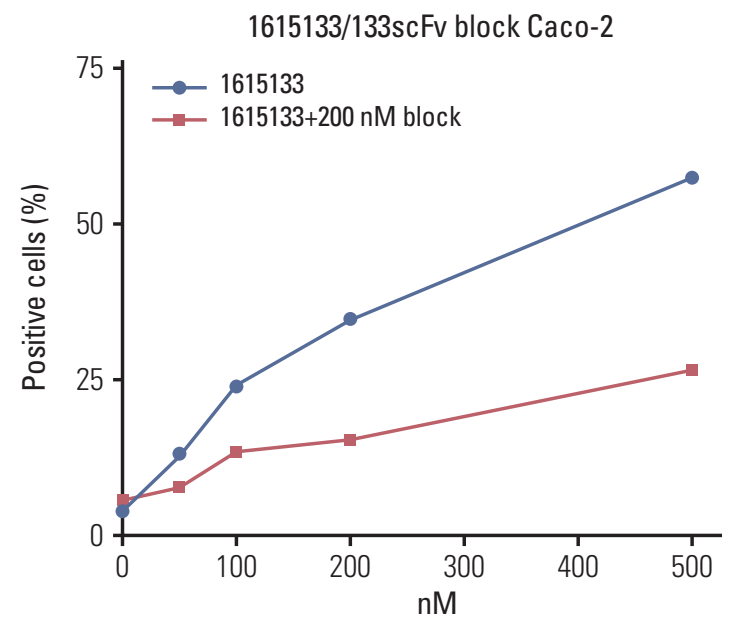

Fig. 4. ${ }^{51} \mathrm{Chromium}$ release and binding. (A, B) To evaluate the drug activity, ${ }^{51} \mathrm{Cr}$ release assays using two donors were performed. 1615133 TriKE, $16133 \mathrm{BiKE}$, anti-CD16 scFv (16scFv), and anti-CD133 scFv (133scFv) (30 nM) were cocultured with CD133 ${ }^{+}$Caco-2 cells and peripheral blood mononuclear cells at the labeled E:T ratios. (C) Peripheral blood mononuclear cells and Caco-2 cells were exposed to different TriKE concentrations (1, 5, and $10 \mathrm{nM}$ ) and titered at their E:T ratio (20:1, 6.6:1, 2.2:1, 0.7:1, 0.23:1, and 0.08:1). (D) Fluorescein isothiocyate labeled 1615133 TriKE was incubated at the labeled concentrations with Caco-2 cells. In the same experiment, the same amount of FITC labeled 1615133 was added with $200 \mathrm{nM}$ of unlabeled monomeric CD133 scFv for blocking. TriKE, trispecific natural killer cell engager; BiKE, bispecific natural killer cell engager; scFV, single chain variable fragment; E, effector; $\mathrm{T}$, target.

showed larger differences in activity with TriKE inducing the most killing. The dose dependent titration of 1615133 TriKE $(1,5$, and $10 \mathrm{nM})$ with a broader spectrum of E:T ratios (20:1, 6.6:1, 2.2:1, 0.7:1, 0.23:1, and 0.08:1) had the highest impact of the drug activity at higher doses (Fig. 4C). To evaluate the specificity of binding, the flow cytometry-based fluorescence intensity was measured after the incubation of Caco-2 cells with FITC labeled 1615133 TriKE at different concentrations $(1,5,10,50,100,200$, and $500 \mathrm{nM})$. When an unlabeled antiCD133 scFv was added in addition to 1615133 TriKE, binding was reduced potently, indicating that 1615133 TriKE is binding target to cells specifically through the interaction with CD133 (Fig. 4D). Experiments were also performed with 100 and $500 \mathrm{nM}$ to ensure reproducibility. Together, these results 
A

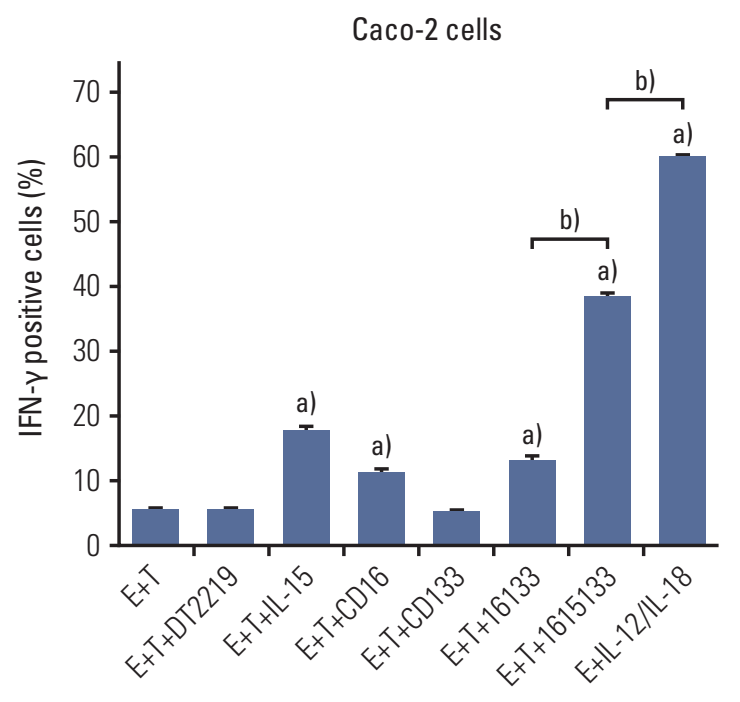

B

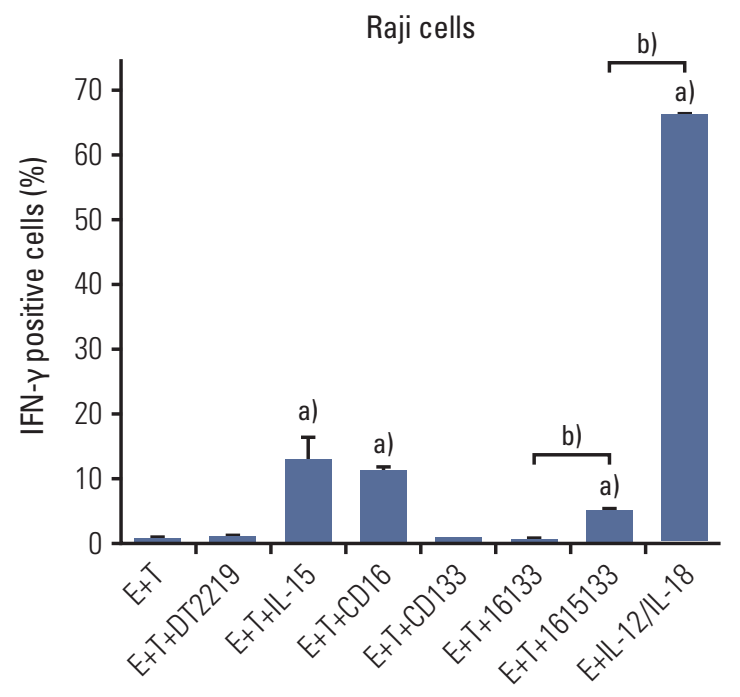

Fig. 5. Intracellular IFN- $\gamma$ production. (A) Peripheral blood mononuclear cells and Caco-2 cell targets were exposed to DT2219, National Cancer Institute derived IL-15 (IL-15), anti-CD16 scFv (CD16), anti-CD133 scFv (CD133), 16133 BiKE, 1615133 TriKE $(50 \mathrm{nM})$, or an IL-12 $(10 \mathrm{ng} / \mathrm{mL})$ and IL-18 $(100 \mathrm{ng} / \mathrm{mL})$ positive control (IL-12/IL-18). The gates were set on $\mathrm{CD}^{2} 6^{+} \mathrm{CD} 3^{-} \mathrm{NK}$ cells. (B) CD133- Raji cells were exposed to the same panel. IFN- $\gamma$, interferon $\gamma$; IL, interleukin; scFV, single chain variable fragment; $\mathrm{BiKE}$, bispecific natural killer cell engager; TriKE, trispecific natural killer cell engager; NK, natural killer; E, effector; $\mathrm{T}$, target. ${ }^{\mathrm{a})}$ Significance compared to $\mathrm{E}+\mathrm{T}(\mathrm{p}<0.001)$, ${ }^{\mathrm{b}}$ Significance direct comparison $(\mathrm{p}<0.001)$.

indicate that the ADCC mediated by the TriKE was antigen directed.

\section{Ability of IFN- $\gamma$ induction}

To determine if 1615133 TriKE can also induce pro-inflammatory cytokine production in NK cells, flow cytometry was performed to evaluate intracellular IFN- $\gamma$ production. The PBMCs of healthy donors were exposed to CD $133^{+}$Caco-2 targets and $50 \mathrm{nM}$ of DT2219, NCI derived IL-15, anti-CD16 scFv, anti-CD133 scFv, 16133 BiKE, 1615133 TriKE, and supraphysiologic levels of IL-12 and IL-18 (as a positive control). IL-15, anti-CD16 scFv, and BiKE induced a small but significant increase in the proportion of IFN- $\gamma$ producing cells compared to the effector+target control. On the other hand, 1615133 TriKE induced a significantly higher proportion of cytokine-producing NK cells compared to all tested drugs, with the exception of the IL-12/IL-18 positive control, which displayed the highest level of cytokine producing NK cells (Fig. 5A). The Burkitt lymphoma Raji cell line, which is $\mathrm{CD}^{-133^{-}}$was used to evaluate the specificity of IFN- $\gamma$ induction. IL-15, anti-CD16 scFv, and 1615133 TriKE showed negligible elevated levels compared to the control with effectors + targets alone. TriKE showed significantly elevated IFN- $\gamma$ levels compared to BiKE, presumably via the IL-15 moiety,

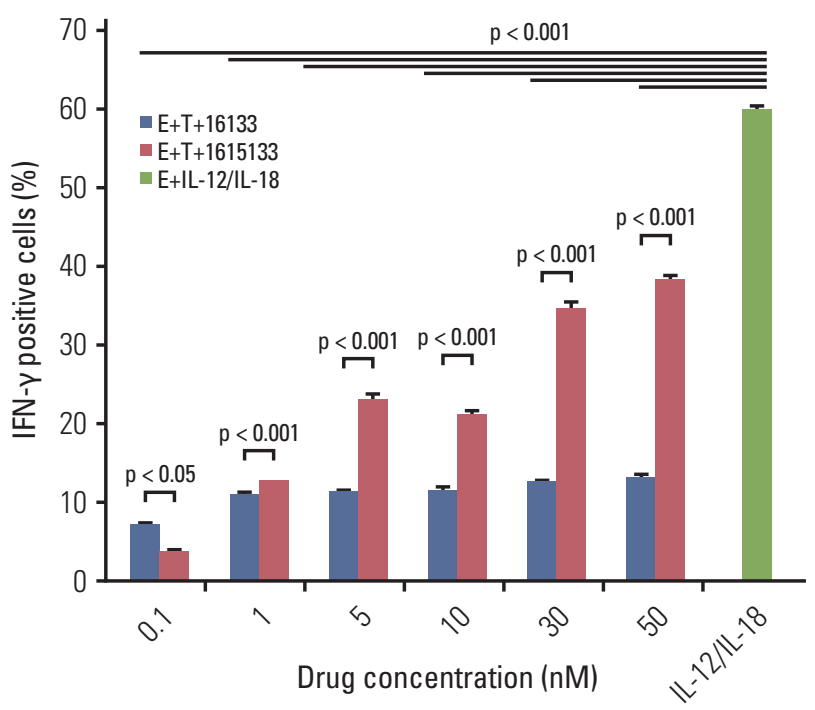

Fig. 6. Dose dependent IFN- $\gamma$ production. Caco-2 cells were incubated with peripheral blood mononuclear cells and exposed to labeled doses of the TriKE and BiKE constructs. After gating on NK cells, flow cytometry showed the respective intracellular IFN- $\gamma$ production. IFN- $\gamma$, interferon $\gamma$; TriKE, trispecific natural killer cell engager; BiKE, bispecific natural killer cell engager; NK, natural killer; E, effector; $\mathrm{T}$, target; $\mathrm{IL}$, interleukin. 
but also significantly lower levels than the IL-12 and IL-18 control (Fig. 5B).

The efficacy of IL-15 in the 16133 scaffold was made more obvious by comparing the dose dependent IFN- $\gamma$ elevation of 16133 BiKE and 1615133 TriKE. PBMCs were cocultured with $\mathrm{CD} 133^{+}$Caco-2 cells and exposed to the BiKE and TriKE in increasing concentrations $(0.1,1,5,10,30$, and $50 \mathrm{nM})$. Superior NK cell-related IFN- $\gamma$ production of TriKE was observed at a minimum of $1 \mathrm{nM}$ and increased in a dose dependent manner, whereas the BiKE group showed constant IFN- $\gamma$ levels that were significantly lower than TriKE. Both drugs showed significantly lower IFN- $\gamma$ levels than the positive IL-12/IL-18 group (Fig. 6).

\section{Discussion}

This study documents the formation and testing of a selfsustaining NK cell engager that targets NK cells and CD133 cells via anti-CD16 and anti-CD133 scFvs. To improve the mode of action of the previously published 16133 BiKE [19], this platform was used to introduce a modified IL-15 cross linker. The new TriKE mediates the specific and improved ADCC and causes NK cell proliferation and prolonged survival. These characteristics were not inducible either with 16133 BiKE, anti-CD16 scFv alone, anti-CD133 scFv alone, or IL-15 alone. In addition, our group previously demonstrated that this same scFv is capable of targeting CD133+ tumor-initiating cells [19]. We have effectively produced the first CSCspecific NK cell immune engager that is capable of simultaneously mediating ADCC and effector cell expansion that can serve as a sufficient off-the-shelf therapy.

Overall, the data suggest that the 1615133 TriKE could theoretically provide an inexpensive alternative to ongoing CAR $\mathrm{T}$ cell approaches. In particular, this molecule possesses characteristics that might better drive the NK cell targeting of CSCs.

The benefits of the combinational use of $\mathrm{mABs}$ and cytokines, such as IL-15 and IL-2, to improve NK cell-based immunotherapy have been hypothesized [24]. The safety profile, however, tends to be beneficial with IL-15 considering the clinical trials that used IL-2 in pediatric patients suffering from neuroblastoma and adults with ovarian carcinoma. The authors reported that besides the minor signs of improved anticancer reactivity, IL-2-related toxicity leads to serious adverse events, resulting in dose-limiting administration [25]. Furthermore, a murine model showed that the dose to induce pulmonary vascular leakage was 6 times higher with IL-15 exposure than with IL-2, supporting a superior safety profile of IL-15 [26]. Several clinical studies are ongoing using IL-15 to treat patients with refractory malignant melanoma and metastatic renal cell cancer or as an adjuvant therapy after chemotherapy and lymphocyte transfer in metastatic melanoma. A phase I study included 12 consecutive patients with metastatic melanoma and renal cell cancer, and IL-15 administration showed a response in five patients with a decrease in their marker lesions. In addition to these results, the authors stated the impact in NK cellrelated anti-cancer performance by the mediation of proliferation and prolonged survival $[27,28]$ implying the valuable impact of IL-15 in NK cell related anti-cancer defense. The advantage of introducing the IL-15 molecule to BiKE rather than combining BiKE with IL-15 was observed in previously studied TriKEs 161533 [14] and 1615EpCAM [20]. A side-by-side comparison between the respective TriKEs with BiKEs plus IL-15 showed that the TriKEs induced superior functionality in various entities compared to the separate administration of IL-15. This indicates a different mode of action. Furthermore, targeting via TriKE might improve the accumulation of the IL-15 stimulus at the site of the malignancy. The expectation with 1615133 TriKE is that this molecule will enhance targeting and killing at the site of the CSC pool through the delivery of an ADCC and IL-15 signal.

On the other hand, side effects also occur with IL-15 administration that can cause serious complications. Conlon et al. [27] reported a hemodynamic imbalance and typical cytokine-related complications, such as fever and chills, restricted to Common Terminology Criteria for Adverse Events (CTCAE) grade 3 toxicity. Myelosupressive disturbances were reported in one of twelve patients with the highest CTCAE grade 4 concerning lymphopenia [27].

Owing to the reported short term IL-6, IL-8, and IFN- $\gamma$ elevations after recombinant human IL-15 administration, this study focused on IFN- $\gamma$ because it is a well-recognized marker cytokine indicating a cytokine storm. Moderate IFN$\gamma$ release after 16133 BiKE exposure has already been recognized [19]. A side-by-side comparison of BiKE and TriKE revealed a dose dependent enhancement of IFN- $\gamma$ release that was higher with the IL-15-containing drug but moderate compared to the supraphysiologic IL-12/IL-18 control. On the other hand, there is the possibility that by targeting NK cells with IL-15, 1615133 TriKE would restrict IL-15 signaling to that subset alone, possibly decreasing the overall negative impact and adverse effects. Furthermore IFN- $\gamma$ has antitumor function and is necessary for inducing a productive inflammatory response [29]. Therefore, the moderate IFN- $\gamma$ release induced by 1615133 TriKE might bolster the anti-cancer effects while reducing the toxicity by targeting IL-15 to NK cells.

The sufficient elimination of CSCs is still an unachieved goal in oncology patients. Characteristics, such as chemoresistance and radio-resistance [1], as well as tumor initiating 
and self-renewal abilities lead to the persistence of CSCs in the patient and represents a known cause for drug refractory relapse, even after years of remission. Studies describe the occurrence of CSCs in several tumor entities, such as breast, colon, prostate, liver, pancreatic, lung cancer, and head and neck squamous cell carcinoma implying the potential for fundamental improvement in cancer treatment by specifically targeting CSCs. The targeting of $\mathrm{CD} 133^{+}$cells leads to a tumor response in breast, ovarian, and gastrointestinal cancer $[30,31]$ even if CSCs represent only a minor percentage $(<10 \%)$ of the entire tumor mass [32]. Similar results have been shown with toxin-containing constructs that sufficiently eliminated $\mathrm{CD} 133^{+} \mathrm{CSC}$ in vitro as well as in a murine model $[30,33]$. These findings suggest that the novel 1615133 TriKE, described here, has the potential to curve relapse by targeting the CSC pool.

Note that the authors used a fluorescently labeled antiCD133 scFv, which is same as the scFv present in 1615133 TriKE, to enrich $\mathrm{CD} 133^{+}$cells via flow cytometric sorting and these cells were used in tumor initiation assays. In an in vivo mouse xenograft model, the enriched cells augmented the size and speed of human head and neck cancer tumor growth [31]. Expedited tumor initiation has been shown to be a hallmark of CSC activity. This data helps validate the targeting of CD133 in TriKE.

CD133 was reported to be expressed in normal stem cells, which are found in the nervous and vascular system as well as in pluripotent stem cells of the hematopoietic system [34-36]. Drug safety analyses in previous studies using a targeted toxin linked to an identical anti-CD133 ${ }^{+} \mathrm{scFv}$ (dCD133KDEL) revealed very little non-target-related toxicity in a murine model and in human in vitro pluripotent stem cell assays [37]. This lack of reactivity to normal stem cells could be attributed to the differential copy number. Even if normal stem cells are being targeted, Rutella et al. [38] described the occurrence of $\mathrm{CD}^{133^{-}}$pluripotent stem cells in bone marrow capable of differentiating into lymphoid, myeloid, and $\mathrm{CD}_{133^{+}}$cells implicating the sufficient regeneration of the hematopoietic system even after the elimination of CD133 stem cells [39].

The incorporation of a modified IL-15 crosslinker in the 16133 BiKE scaffold forming 1615133 TriKE shows therapeutic promise. The new TriKE demonstrated an improvement of ADCC. Furthermore, the focused delivery of IL-15 stimulus as part of the same molecule that harnesses NK cells to form an immunological synapse with cancer cells provides prolonged survival and proliferation of effectors. The data suggests that the new construct might address the need to provide a highly active immune engager capable of eliminating CSCs at the root of drug refractory relapse in cancer patients.

\section{Conflicts of Interest}

Drs. Vallera and Miller are members of the Oxis Biotech Scientific Advisory Board and hold equity in the company. This relationship has been reviewed and managed by the University of Minnesota in accordance with its conflict of interest policies.

\section{Acknowledgments}

This work was supported in part by the US Public Health Service Grant R01-CA36725, R01-CA72669, P01-CA65493, P01-CA111412 and R35 CA197292 awarded by the NCI and the NIAID, DHHS. It was also supported by an NIH Research Evaluation and Commercialization Hub (REACH) Award (U01), the Randy Shaver Cancer Research and Community Foundation, the Atwater Cancer Drug Development Award, the Minnesota Ovarian Cancer Alliance (MOCA) the Deutsche Krebshilfe (J.U.S., 111548), and a CETI translational award from the University of Minnesota Masonic Cancer Center.

\section{References}

1. Kim Y, Joo KM, Jin J, Nam DH. Cancer stem cells and their mechanism of chemo-radiation resistance. Int J Stem Cells. 2009;2:109-14.

2. Du Z, Qin R, Wei C, Wang M, Shi C, Tian R, et al. Pancreatic cancer cells resistant to chemoradiotherapy rich in "stem-celllike" tumor cells. Dig Dis Sci. 2011;56:741-50.

3. Fabrizi E, di Martino S, Pelacchi F, Ricci-Vitiani L. Therapeutic implications of colon cancer stem cells. World J Gastroenterol. 2010;16:3871-7.

4. Smith LM, Nesterova A, Ryan MC, Duniho S, Jonas M, Anderson M, et al. CD133/ prominin-1 is a potential therapeutic tar- get for antibody-drug conjugates in hepatocellular and gastric cancers. Br J Cancer. 2008;99:100-9.

5. Hashimoto K, Aoyagi K, Isobe T, Kouhuji K, Shirouzu K. Expression of CD133 in the cytoplasm is associated with cancer progression and poor prognosis in gastric cancer. Gastric Cancer. 2014;17:97-106.

6. Carson WE, Giri JG, Lindemann MJ, Linett ML, Ahdieh M, Paxton R, et al. Interleukin (IL) 15 is a novel cytokine that activates human natural killer cells via components of the IL-2 receptor. J Exp Med. 1994;180:1395-403.

7. Fehniger TA, Shah MH, Turner MJ, VanDeusen JB, Whitman 
SP, Cooper MA, et al. Differential cytokine and chemokine gene expression by human NK cells following activation with IL-18 or IL-15 in combination with IL-12: implications for the innate immune response. J Immunol. 1999;162:4511-20.

8. Cooper MA, Fehniger TA, Turner SC, Chen KS, Ghaheri BA, Ghayur T, et al. Human natural killer cells: a unique innate immunoregulatory role for the CD56(bright) subset. Blood. 2001;97:3146-51.

9. Childs RW, Berg M. Bringing natural killer cells to the clinic: ex vivo manipulation. Hematology Am Soc Hematol Educ Program. 2013;2013:234-46.

10. Fehniger TA, Cooper MA, Caligiuri MA. Interleukin-2 and interleukin-15: immunotherapy for cancer. Cytokine Growth Factor Rev. 2002;13:169-83.

11. Singer H, Kellner C, Lanig H, Aigner M, Stockmeyer B, Oduncu F, et al. Effective elimination of acute myeloid leukemic cells by recombinant bispecific antibody derivatives directed against CD33 and CD16. J Immunother. 2010;33: 599-608.

12. Stein C, Kellner C, Kugler M, Reiff N, Mentz K, Schwenkert $\mathrm{M}$, et al. Novel conjugates of single-chain Fv antibody fragments specific for stem cell antigen CD123 mediate potent death of acute myeloid leukaemia cells. Br J Haematol. 2010; 148:879-89.

13. Swaminathan SK, Niu L, Waldron N, Kalscheuer S, Zellmer DM, Olin MR, et al. Identification and characterization of a novel scFv recognizing human and mouse CD133. Drug Deliv Transl Res. 2013;3:143-51.

14. Vallera DA, Felices M, McElmurry R, McCullar V, Zhou X, Schmohl JU, et al. IL15 trispecific killer engagers (TriKE) make natural killer cells specific to CD33+ targets while also inducing persistence, in vivo expansion, and enhanced function. Clin Cancer Res. 2016;22:3440-50.

15. Plosker GL, Figgitt DP. Rituximab: a review of its use in nonHodgkin's lymphoma and chronic lymphocytic leukaemia. Drugs. 2003;63:803-43.

16. Schmohl JU, Vallera DA. CD133, selectively targeting the root of cancer. Toxins (Basel). 2016;8:E165.

17. McCall AM, Adams GP, Amoroso AR, Nielsen UB, Zhang L, Horak E, et al. Isolation and characterization of an anti-CD16 single-chain Fv fragment and construction of an anti-HER2/ neu/anti-CD16 bispecific scFv that triggers CD16-dependent tumor cytolysis. Mol Immunol. 1999;36:433-45.

18. Zhu X, Marcus WD, Xu W, Lee HI, Han K, Egan JO, et al. Novel human interleukin-15 agonists. J Immunol. 2009;183: 3598-607.

19. Schmohl JU, Gleason MK, Dougherty PR, Miller JS, Vallera DA. Heterodimeric bispecific single chain variable fragments $(\mathrm{scFv})$ killer engagers (BiKEs) enhance NK-cell activity against CD133+ colorectal cancer cells. Target Oncol. 2016;11:353-61.

20. Schmohl JU, Felices M, Taras E, Miller JS, Vallera DA. Enhanced ADCC and NK Cell activation of an anticarcinoma bispecific antibody by genetic insertion of a modified IL-15 cross-linker. Mol Ther. 2016;24:1312-22.

21. Fogh J, Fogh JM, Orfeo T. One hundred and twenty-seven cultured human tumor cell lines producing tumors in nude mice.
J Natl Cancer Inst. 1977;59:221-6.

22. Roederer M. Interpretation of cellular proliferation data: avoid the panglossian. Cytometry A. 2011;79:95-101.

23. Gleason MK, Verneris MR, Todhunter DA, Zhang B, McCullar $\mathrm{V}$, Zhou SX, et al. Bispecific and trispecific killer cell engagers directly activate human NK cells through CD16 signaling and induce cytotoxicity and cytokine production. Mol Cancer Ther. 2012;11:2674-84.

24. Childs RW, Carlsten M. Therapeutic approaches to enhance natural killer cell cytotoxicity against cancer: the force awakens. Nat Rev Drug Discov. 2015;14:487-98.

25. Connor JP, Felder M, Hank J, Harter J, Gan J, Gillies SD, et al. Ex vivo evaluation of anti-EpCAM immunocytokine huKS-IL2 in ovarian cancer. J Immunother. 2004;27:211-9.

26. Munger W, DeJoy SQ, Jeyaseelan R Sr, Torley LW, Grabstein $\mathrm{KH}$, Eisenmann J, et al. Studies evaluating the antitumor activity and toxicity of interleukin-15, a new T cell growth factor: comparison with interleukin-2. Cell Immunol. 1995;165: 289-93.

27. Conlon KC, Lugli E, Welles HC, Rosenberg SA, Fojo AT, Morris JC, et al. Redistribution, hyperproliferation, activation of natural killer cells and CD8 T cells, and cytokine production during first-in-human clinical trial of recombinant human interleukin-15 in patients with cancer. J Clin Oncol. 2015;33: 74-82.

28. Waldmann TA. Interleukin-15 in the treatment of cancer. Expert Rev Clin Immunol. 2014;10:1689-701.

29. Ikeda H, Old LJ, Schreiber RD. The roles of IFN gamma in protection against tumor development and cancer immunoediting. Cytokine Growth Factor Rev. 2002;13:95-109.

30. Waldron NN, Barsky SH, Dougherty PR, Vallera DA. A bispecific EpCAM/CD133-targeted toxin is effective against carcinoma. Target Oncol. 2014;9:239-49.

31. Waldron NN, Kaufman DS, Oh S, Inde Z, Hexum MK, Ohlfest JR, et al. Targeting tumor-initiating cancer cells with dCD133KDEL shows impressive tumor reductions in a xenotransplant model of human head and neck cancer. Mol Cancer Ther. 2011;10:1829-38.

32. Ohlfest JR, Zellmer DM, Panyam J, Swaminathan SK, Oh S, Waldron NN, et al. Immunotoxin targeting CD133(+) breast carcinoma cells. Drug Deliv Transl Res. 2013;3:195-204.

33. Damek-Poprawa M, Volgina A, Korostoff J, Sollecito TP, Brose MS, O'Malley BW Jr, et al. Targeted inhibition of CD133+ cells in oral cancer cell lines. J Dent Res. 2011;90:638-45.

34. Yin AH, Miraglia S, Zanjani ED, Almeida-Porada G, Ogawa M, Leary AG, et al. AC133, a novel marker for human hematopoietic stem and progenitor cells. Blood. 1997;90: 5002-12.

35. Peichev M, Naiyer AJ, Pereira D, Zhu Z, Lane WJ, Williams $\mathrm{M}$, et al. Expression of VEGFR-2 and AC133 by circulating human CD34(+) cells identifies a population of functional endothelial precursors. Blood. 2000;95:952-8.

36. Uchida N, Buck DW, He D, Reitsma MJ, Masek M, Phan TV, et al. Direct isolation of human central nervous system stem cells. Proc Natl Acad Sci U S A. 2000;97:14720-5.

37. Skubitz AP, Taras EP, Boylan KL, Waldron NN, Oh S, 
Panoskaltsis-Mortari A, et al. Targeting CD133 in an in vivo ovarian cancer model reduces ovarian cancer progression. Gynecol Oncol. 2013;130:579-87.

38. Rutella S, Bonanno G, Marone M, De Ritis D, Mariotti A, Voso $\mathrm{MT}$, et al. Identification of a novel subpopulation of human cord blood CD34-CD133-CD7-CD45+lineage- cells capable of
lymphoid/NK cell differentiation after in vitro exposure to IL-15. J Immunol. 2003;171:2977-88.

39. Suuronen EJ, Wong S, Kapila V, Waghray G, Whitman SC, Mesana TG, et al. Generation of CD133+ cells from CD133peripheral blood mononuclear cells and their properties. Cardiovasc Res. 2006;70:126-35. 